\section{Endoscopic removal of migrating silastic band after vertical banding gastroplasty}

A 33-year-old woman was investigated for persistent dysphagia, severe gastroesophageal reflux disease, and refractory vomiting. She had undergone vertical banding gastroplasty (VBG) 5 years earlier to manage her obesity and had successfully lost $90 \mathrm{~kg}$ in weight. This was performed following failed laparoscopic adjustable gastric banding (LAGB), in which significant reflux symptoms required deflation of the band. Physical examination was normal. Upper gastrointestinal endoscopy revealed a gastric pouch at $35-46 \mathrm{~cm}$ from the incisors, which contained residual food particles. Within the gastric lumen, migration of a silastic band from the VBG was noted ( $\bullet$ Fig. $\mathbf{1}$ ).

The band was removed under endoscopic guidance with a dual-channel therapeutic endoscope. The band was grasped with an alligator forceps and, by utilizing the second endoscopic channel, an endoloop cutter was employed to cut the band. Traction was maintained with the forceps until the band was cut and successfully retrieved ( $\bullet$ Figs. 2 and 3 ; $\bullet$ Video 1 ).

Obesity has become a major public health issue worldwide - a chronic illness that engenders multiple obesity-related comorbidities. Present in $18 \%$ of adolescents and $23 \%$ of adults, its prevalence is on the rise. Prior to the advent of LAGB, VBG was considered a standard surgical option for the management of obesity. It involves the formation of a proximal gastric pouch through the insertion of vertical staples and placement of an external prosthetic band, which provides support. Food has

\section{Video 1}

Endoscopic removal of migrated silastic band from vertical banding gastroplasty. to pass through this narrow band; hence the weight loss mechanism is via a restrictive process. This approach is now less favored due to the high incidence of complications and lack of sustained weight loss. Part of the appeal of the LAGB technique is its adjustability and reversibility. VBG however may be considered as a secondline option. Complications include nausea and vomiting, gastroesophageal reflux disease, staple line disruption, band erosion, marginal ulcers, and weight re-gain. Migrated silastic bands can be safely removed endoscopically as demonstrated in this patient, thus avoiding the need for further surgical intervention.

\section{Competing interests: None}

Endoscopy_UCTN_Code_TTT_1AO_2AD

\section{S. G. Chen Yi Mei, W. Tam, G. Nind, R. Singh}

Department of Gastroenterology, Lyell McEwin Hospital, Elizabeth Vale, South Australia, Australia

\section{Bibliography}

DOI $10.1055 / \mathrm{s}-0030-1255643$

Endoscopy 2010; 42: E253

(c) Georg Thieme Verlag KG Stuttgart · New York . ISSN 0013-726X

\section{Corresponding author \\ S. G. Chen Yi Mei, MBBS}

Department of Gastroenterology

Lyell McEwin Hospital

Haydown Rd

Elizabeth Vale

5112 South Australia

Australia

Fax: +61-8-1829837

sweelinc@gmail.com

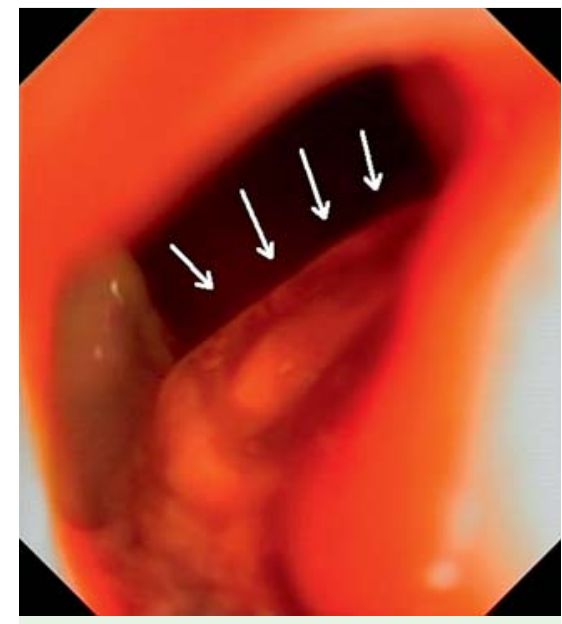

Fig. 1 Silastic band migrating into gastric lumen.

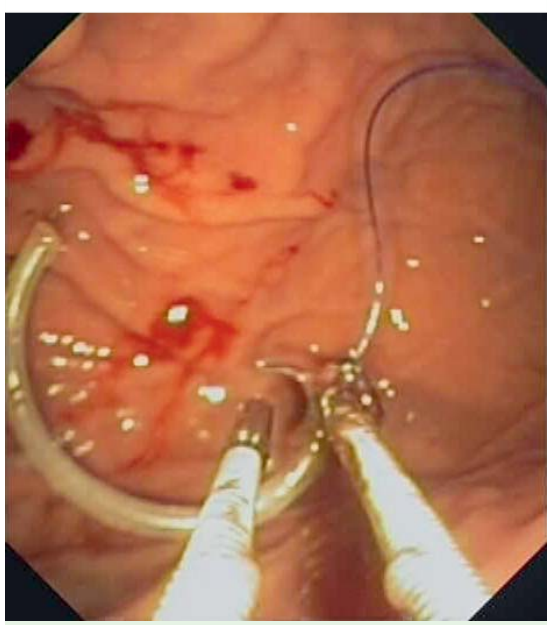

Fig. 2 Silastic band retrieved by utilizing an alligator forceps and endoloop cutter.

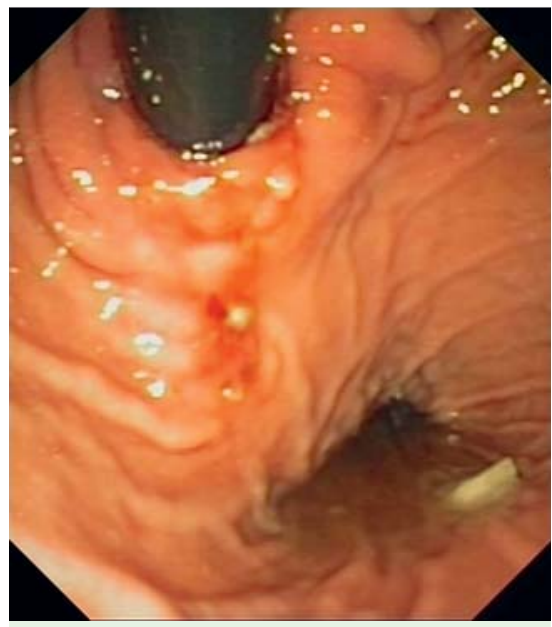

Fig. 3 Silastic band removed from the gastric lumen, with the perforation site evident below the endoscope. 